\title{
An extension of Stein-Lovász theorem and some of its applications
}

\author{
Guang-Siang Lee
}

Published online: 5 October 2011

(C) Springer Science+Business Media, LLC 2011

\begin{abstract}
The Stein-Lovász theorem provides an algorithmic way to deal with the existence of certain good coverings, and thus offers bounds related to some combinatorial structures. An extension of the classical Stein-Lovász theorem for multiple coverings is given, followed by some applications for finding upper bounds of the sizes of $(d, s$ out of $r ; z]$-disjunct matrices and $(k, m, c, n ; z)$-selectors, respectively. This gives a unified treatment for some previously known results relating to various models of group testing.
\end{abstract}

Keywords Stein-Lovász theorem · Disjunct matrices $\cdot$ Selectors

\section{Introduction}

Let $X$ be a finite set and $\Gamma$ be a family of subsets of $X$. Denote by $H=(X, \Gamma)$ the hypergraph having $X$ as the set of vertices and $\Gamma$ as the set of hyperedges. A subset $T \subseteq X$ such that $T \cap E \neq \emptyset$ for any hyperedge $E$ is called a vertex cover (synonymously: transversal or hitting set) of the hypergraph $H$. The minimum size of a vertex cover of the hypergraph $H$ is denoted by $\tau(H)$. An upper bound for $\tau(H)$ was given by Lovász (1975):

$$
\tau(H)<\frac{|X|}{\min _{E \in \Gamma}|E|}(1+\ln \Delta),
$$

where $\Delta=\max _{x \in X} \mid\{E: E \in \Gamma$ and $x \in E\} \mid$. An equivalent statement in terms of the point-block incidence matrices of the corresponding hypergraphs was given by Stein (1974) independently. It was called the Stein-Lovász Theorem in Cohen et al. (1996)

G.-S. Lee ( $\square)$

Department of Applied Mathematics, National Chiao-Tung University, Hsinchu 30010, Taiwan e-mail: tremolo.am96g@nctu.edu.tw 
while dealing with the covering problems in coding theory, see Sect. 2 for further details.

The Stein-Lovász theorem was used in dealing with the upper bounds for the sizes of $(k, m, n)$-selectors (De Bonis et al. 2005), followed by the upper bounds for the sizes of $(d, r ; z]$-disjunct matrices (Chen et al. 2008). More of its applications for treating the minimal sizes of various set systems were also found in Deng et al. (2011). These applications were obtained by translating the problems into the hypergraph problems and showing that the models can be deduced from the vertex cover of properly defined hypergraphs. The notion of $(k, m, n)$-selectors was first introduced by De Bonis et al. (2005), followed by a generalization to the notion of $(k, m, w, n)$ selectors (De Bonis 2008). In this paper, we use the notation $(k, m, c, n)$-selectors for convenience. Further generalizations of $(d, r ; z]$-disjunct matrices and $(k, m, c, n)$ selectors to $(d, s$ out of $r ; z]$-disjunct matrices and $(k, m, c, n ; z)$-selectors respectively will be given in Sect. 2. The variable $z$ here is for error-tolerance purpose in the group testing application (see Definition 2.7 and the following paragraph). A notorious feature of biological experiments is that errors almost always occur during the testing procedure. Therefore, it would be wise for group testing to allow some outcomes to be affected by errors.

In order to deal with the upper bounds for these binary matrices defined in Sect. 2, an extended Stein-Lovász theorem is derived in Sect. 3, which can be regarded as a more general version in terms of " $z$-cover", i.e., a subset $T$ of vertices such that each hyperedge contains at least $z$ elements in $T$. Although there is an intuitive way to form a $z$-cover by making $z$ copies of all elements of a vertex cover, the $z$-cover we construct consists of distinct elements. This extension can be useful practically, for example, in experiment designs all experiments are usually required to be distinct because duplicates of experiments are redundant and meaningless.

In Sect. 4, the extended Stein-Lovász theorem will be used in dealing with the upper bounds for the sizes of $(d, s$ out of $r ; z]$-disjunct matrices (Theorem 4.1) and $(k, m, c, n ; z)$-selectors (Theorem 4.3), respectively. It in turn provides a few upper bounds for the sizes in various models with specific parameters as shown in Corollary 4.2 and Corollary 4.4.

\section{Preliminaries}

\subsection{Equivalence of the theorems of Lovász and Stein}

For the hypergraph $H=(X, \Gamma)$ with the vertex set $X$ and the hyperedge set $\Gamma$, the degree of $x \in X$ is the number of hyperedges containing $x$, and $\Delta$ denotes the maximum degree in $H$. A binary matrix $M=\left(m_{E_{i} x_{j}}\right)$ of order $|\Gamma| \times|X|$ can be interpreted as a block-point incidence matrix of the hypergraph $H$, i.e., the rows of $M$ correspond to the hyperedge set $\Gamma=\left\{E_{1}, E_{2}, \ldots, E_{|\Gamma|}\right\}$, and the columns correspond to the vertex set $X=\left\{x_{1}, x_{2}, \ldots, x_{|X|}\right\}$, where

$$
m_{E_{i} x_{j}}= \begin{cases}1 & \text { if the hyperedge } E_{i} \text { contains the vertex } x_{j} \\ 0 & \text { otherwise. }\end{cases}
$$


A subset $N \subseteq \Gamma$ (the same hyperedge may occur more than once) such that each vertex belongs to at most $z$ of its members is called a $z$-matching of the hypergraph $H$. The maximum size over all $z$-matchings of the hypergraph $H$ is denoted by $v_{z}(H)$. Thus $v(H)=v_{1}(H)$ is the maximum number of disjoint hyperedges. For $v_{z}(H)$ and other functions to be defined we often remove the argument $H$ when the context is clear. A $z$-matching is simple if no hyperedge occurs in it more than once. Let $\widetilde{v}_{z}$ be the maximum number of hyperedges in simple $z$-matchings. Clearly, $\widetilde{v}_{z} \leq v_{z}$. A subset $T \subseteq X$ (the same vertex does not occur more than once) such that $|T \cap E| \geq z$ for any hyperedge $E$ is called a $z$-cover of the hypergraph $H$. Note that the requirement "the same vertex does not occur more than once" is different from the definition of the origional $z$-cover in Lovász (1975). The minimum size over all $z$-covers of the hypergraph $H$ is denoted by $\tau_{z}(H)$. Thus $\tau(H)=\tau_{1}(H)$ is the minimum size of a vertex cover of the hypergraph $H$.

A vector $\left(w_{E_{1}}, w_{E_{2}}, \ldots, w_{E_{|\Gamma|}}\right)$ with $w_{E_{i}} \geq 0$ for each $E_{i} \in \Gamma$ is called a fractional matching of the hypergraph $H$ if each entry of the vector $\left(w_{E_{1}}, w_{E_{2}}, \ldots, w_{E_{|\Gamma|}}\right) M$ is at most 1 . A vector $\left(w_{x_{1}}, w_{x_{2}}, \ldots, w_{x_{|X|}}\right)$ with $w_{x_{i}} \geq 0$ for each $x_{i} \in X$ is called a fractional cover of the hypergraph $H$ if each entry of the vector $M\left(w_{x_{1}}, w_{x_{2}}, \ldots, w_{x_{|X|}}\right)^{t}$ is at least 1 . Define

$$
v^{*}(H)=\sup \sum_{E_{i} \in \Gamma} w_{E_{i}} \text { and } \tau^{*}(H)=\inf \sum_{x_{i} \in X} w_{x_{i}},
$$

where the extrema are taken over all fractional matchings $\left(w_{E_{1}}, w_{E_{2}}, \ldots, w_{E_{|\Gamma|}}\right)$ and all fractional covers $\left(w_{x_{1}}, w_{x_{2}}, \ldots, w_{x_{|X|}}\right)$, respectively. By the duality theorem of linear programming, we have $v^{*}=\tau^{*}$. Then it is easy to see that

$$
v \leq v_{z} / z \leq v^{*}=\tau^{*} \leq \tau_{z} / z
$$

One of the most natural methods to produce a small vertex cover of a given hypergraph $H$ is the "Greedy Cover Algorithm", which we describe as follows (Lovász 1975):

1. Let $x_{1}$ be a vertex with maximum degree.

2. Suppose that $x_{1}, x_{2}, \ldots, x_{i}$ have been already selected. If $x_{1}, x_{2}, \ldots, x_{i}$ cover all hyperedges, then stop; otherwise let $x_{i+1}$ be a vertex which covers the largest number of uncovered hyperedges.

Generally, the greedy cover algorithm is not the best, but we can expect that it gives a rather good estimate for the upper bound of $\tau(H)$. By the greedy cover algorithm, an upper bound for $\tau(H)$ was given by Lovász (1975).

Theorem 2.1 (Lovász 1975) If $H$ is a hypergraph and the above greedy cover algorithm produces $t$ covering vertices, then

$$
t \leq \frac{\tilde{v}_{1}}{1 \times 2}+\frac{\tilde{v}_{2}}{2 \times 3}+\cdots+\frac{\tilde{v}_{\Delta-1}}{(\Delta-1) \times \Delta}+\frac{\widetilde{v}_{\Delta}}{\Delta} .
$$


Corollary 2.2 (Lovász 1975) For a hypergraph H,

$$
\tau(H) \leq\left(1+\frac{1}{2}+\cdots+\frac{1}{\Delta}\right) \tau^{*}(H)<(1+\ln \Delta) \tau^{*}(H) .
$$

Theorem 2.3 (Lovász 1975) For a hypergraph $H=(X, \Gamma)$,

$$
\tau(H)<\frac{|X|}{\min _{E \in \Gamma}|E|}(1+\ln \Delta) .
$$

Similarly, by the greedy cover algorithm, an equivalent statement in terms of the point-block incidence matrices of the corresponding hypergraphs was given by Stein (1974) independently.

Theorem 2.4 (Stein 1974) Let $X$ be a finite set of cardinality $n$, and let $\Gamma=\left\{A_{1}, A_{2}\right.$, $\left.\ldots, A_{m}\right\}$ be a family of subsets of $X$, where $\left|A_{i}\right| \leq$ a for all $1 \leq i \leq m$. Assume that each element of $X$ is in at least $v$ members of the set $\Gamma$. Then there is a subfamily of $\Gamma$ that covers $X$ and has at most

$$
\frac{n}{a}+\frac{m}{v}\left(\frac{1}{2}+\frac{1}{3}+\cdots+\frac{1}{a}\right)
$$

members.

Note that Theorem 2.4 is closely related to the work of Fulkerson and Ryser (1963) in the 1-width of a $(0,1)$-matrix. They define the 1-width of such a matrix, $A$, as the minimum number of columns that can be selected from $A$ in such a way that each row of the resulting submatrix has at least one 1 . In this terminology, Theorem 2.4 can be restated as follows:

Theorem 2.5 (Stein 1974) Let A be a $(0,1)$-matrix with $n$ rows and $m$ columns. Assume that each row contains at least $v$ ones and each column at most a ones. Then the 1-width of $A$ is at most

$$
\frac{n}{a}+\frac{m}{v}\left(\frac{1}{2}+\frac{1}{3}+\cdots+\frac{1}{a}\right)
$$

Theorem 2.5 was called the Stein-Lovász Theorem in Cohen et al. (1996) while dealing with the covering problems in coding theory. The Stein-Lovász theorem was used in dealing with the upper bounds for the sizes of $(k, m, n)$-selectors (De Bonis et al. 2005). Inspired by this work, it was also used in dealing with the upper bounds for the sizes of $(d, r ; z]$-disjunct matrices (Chen et al. 2008). Some more applications can also be found in Deng et al. (2011).

\subsection{The Stein-Lovász theorem}

We now give a "weighted" version of the Stein-Lovász theorem. The proof is included for completeness. In a binary matrix, the weight of a row (or a column), is the number of entries equal to one. 
Theorem 2.6 (Deng et al. 2011) Let $A$ be a $(0,1)$ matrix with $N$ rows and $M$ columns. Assume that each row contains at least $v$ ones, and each column at most a ones. Then there exists an $N \times K$ submatrix $C$ with

$$
K \leq\left(\frac{N}{a}\right)+\left(\frac{M}{v}\right) \ln a \leq\left(\frac{M}{v}\right)(1+\ln a),
$$

such that $C$ does not contain an all-zero row.

Proof A constructive approach for producing $C$ is presented. Let $A_{a}=A$. We begin by picking the maximal number $K_{a}$ of columns from $A_{a}$, whose supports are pairwise disjoint and each column having $a$ ones (perhaps, $K_{a}=0$ ). Discarding these columns and all rows incident to one of them, we are left with a $k_{a} \times\left(M-K_{a}\right)$ matrix $A_{a-1}$, where $k_{a}=N-a K_{a}$. Clearly, the columns of $A_{a-1}$ have at most $a-1$ ones (indeed, otherwise such a column could be added to the previously discarded set, contradicting its maximality). Now we remove from $A_{a-1}$ a maximal number $K_{a-1}$ of columns having $a-1$ ones and whose supports are pairwise disjoint, thus getting a $k_{a-1} \times$ $\left(M-K_{a}-K_{a-1}\right)$ matrix $A_{a-2}$, where $k_{a-1}=N-a K_{a}-(a-1) K_{a-1}$. The process will terminate after at most $a$ steps. The union of the columns of the discarded sets form the desired submatrix $C$ with $K=\sum_{i=1}^{a} K_{i}$.

The first step of the algorithm gives $k_{a}=N-a K_{a}$, which we rewrite, setting $k_{a+1}=N$, as $K_{a}=\left(k_{a+1}-k_{a}\right) / a$. Analogously, $K_{i}=\left(k_{i+1}-k_{i}\right) / i, 1 \leq i \leq a$. Now we derive an upper bound for $k_{i}$ by counting the number of ones in $A_{i-1}$ in two ways: every row of $A_{i-1}$ contains at least $v$ ones, and every column at most $i-1$ ones, thus

$$
v k_{i} \leq(i-1)\left(M-K_{a}-\cdots-K_{i}\right) \leq(i-1) M .
$$

Furthermore,

$$
\begin{aligned}
K & =\sum_{i=1}^{a} K_{i}=\sum_{i=1}^{a} \frac{k_{i+1}-k_{i}}{i} \\
& =\frac{k_{a+1}}{a}+\frac{k_{a}}{a(a-1)}+\frac{k_{a-1}}{(a-1)(a-2)}+\cdots+\frac{k_{2}}{2 \times 1}-k_{1} \\
& \leq(N / a)+(M / v)(1 / a+1 /(a-1)+\cdots+1 / 2),
\end{aligned}
$$

thus giving the result.

The greedy procedure as shown in the proof constructs the desired submatrix one column at a time, and hence the Algorithm follows (Deng et al. 2011).

Note that the Algorithm shows that some rows of $C$ have weight exactly one. This is the key why the Stein-Lovász theorem can be extended. If the entries of 1 occur in a binary matrix $A$ of order $N \times M$ as uniformly as possible, for example, each row of $A$ contains exactly $v$ ones and each column exactly $a$ ones, then an upper bound for $K$ is found so that the submatrices of order $N \times K$ will share similar property too. 


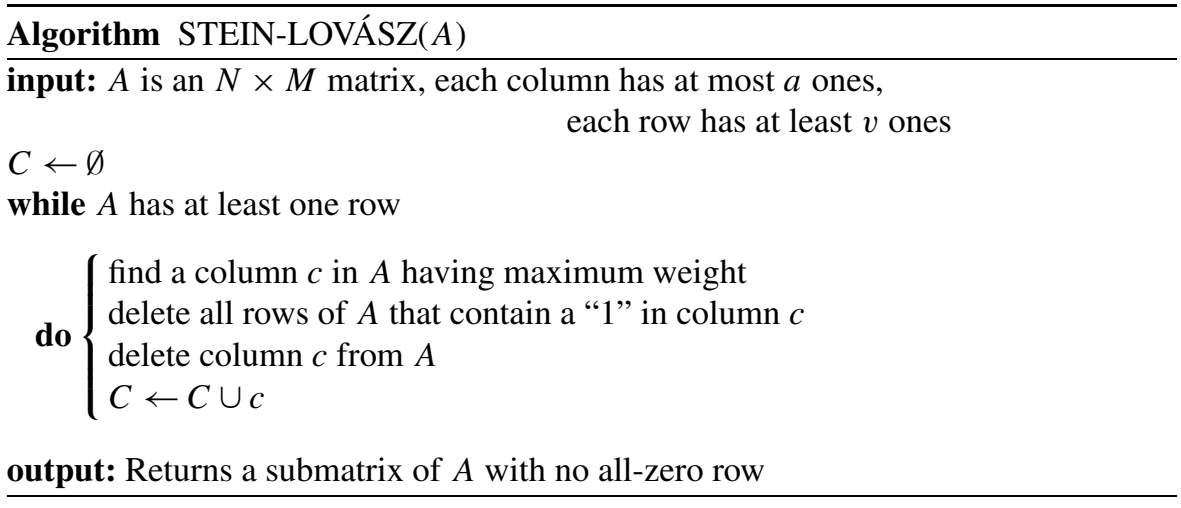

\subsection{A few models for group testing purpose}

A few types of binary matrices will be introduced in this subsection, followed by corresponding associated parameters. These families of binary matrices will be used as models for pooling designs.

Definition 2.7 For integers $d, s, r$ and $z$, with $1 \leq s \leq r$, a $t \times n$ binary matrix $M$ is called $(d, s$ out of $r ; z]$-disjunct if for any $d$ columns and any other $r$ columns of $M$, there exist $z$ row indices in which none of the $d$ columns appear (with entries " 0 ") and at least $s$ of the $r$ columns do (with entries " 1 "). The integer $t$ is the size of the $(d, s$ out of $r ; z]$-disjunct matrix. The minimum size over all $(d, s$ out of $r ; z]$-disjunct matrices with $n$ columns is denoted by $t(n, d, r, s ; z]$.

Note that the notions of $d$-disjunct matrix and $(d ; z)$-disjunct matrix (Cheng and $\mathrm{Du} 2008)$ are equivalent to $(d, 1$ out of $1 ; 1]$-disjunct matrix and $(d, 1$ out of $1 ; z]$-disjunct matrix, respectively. Furthermore, $d$-disjunct and $(d ; z)$-disjunct matrices form the basis for error-free and error-tolerant nonadaptive group testing algorithms (Cheng and Du 2008).

Definition 2.8 For integers $k, m, c$ and $n$ with $1 \leq c<k \leq n$ and $1 \leq m \leq\left(\begin{array}{l}k \\ c\end{array}\right)$, a $t \times n$ binary matrix $M$ is called a $(k, m, c, n ; z)$-selector if any $t \times k$ submatrix of $M$ contains $z$ disjoint submatrices of order $m \times k$ such that in each of them the $m$ rows are all distinct and each row has exactly $c$ entries equal to 1 . The integer $t$ is the size of the $(k, m, c, n ; z)$-selector. The minimum size over all $(k, m, c, n ; z)$-selectors is denoted by $t_{s}(k, m, c, n ; z)$.

It is interesting to remark that the notions of $(k, m, n)$-selectors (De Bonis et al. $2005)$ and $(k, m, c, n)$-selectors (De Bonis 2008) are equivalent to $(k, m, 1, n ; 1)$ selectors and $(k, m, c, n ; 1)$-selectors, respectively. The upper bounds for the size of $(k, m, n)$-selectors and $(k, m, c, n)$-selectors were studied in De Bonis et al. (2005) and in De Bonis (2008) respectively by the Stein-Lovász theorem. The bound for the size of $(k, m, c, n ; z)$-selectors will be derived by the extended Stein-Lovász theorem (Theorem 3.1) in Sect. 4. Table 1 is subclasses of $(d, s$ out of $r ; z]$-disjunct matrices and of $(k, m, c, n ; z)$-selectors, respectively. 
Table 1 Subclasses of ( $d, s$ out of $r ; z]$-disjunct matrices and of $(k, m, c, n ; z)$-selectors

\begin{tabular}{llll}
\hline Parameters & Types & Bounds & References \\
\hline$s=r=1, z=1$ & $d$-disjunct & $t(n, d)$ & Yeh (2002) \\
$s=r=1$ & $(d ; z]$-disjunct & $t(n, d ; z]$ & \\
$s=r, z=1$ & $(d, r]$-disjunct & $t(n, d, r]$ & Yu (2007) \\
$s=r$ & $(d, r ; z]$-disjunct & $t(n, d, r ; z]$ & Chen et al. (2008) \\
$s=1, z=1$ & $(d, r)$-disjunct & $t(n, d, r)$ & Yu (2007) \\
$s=1$ & $(d, r ; z)$-disjunct & $t(n, d, r ; z)$ & \\
$z=1$ & $(d, s$ out of $r]$-disjunct & $t(n, d, r, s]$ & Yu (2007) \\
& $(d, s$ out of $r ; z]$-disjunct & $t(n, d, r, s ; z]$ & \\
$c=1, z=1$ & $(k, m, n)$-selectors & $t_{s}(k, m, n)$ & De Bonis et al. (2005), Yu (2007) \\
$c=1$ & $(k, m, n ; z)$-selectors & $t_{s}(k, m, n ; z)$ & \\
$z=1$ & $(k, m, c, n)$-selectors & $t_{s}(k, m, c, n)$ & De Bonis (2008) \\
& $(k, m, c, n ; z)$-selectors & $t_{s}(k, m, c, n ; z)$ & \\
\hline
\end{tabular}

\subsection{Some basic counting results}

The Stein-Lovász theorem and its extension will be used to estimate the upper bounds of the sizes of various models for pooling designs. In order to give upper bounds for the above mentioned parameters, the following results involving binomial coefficients will be involved. Lemma 2.9 will be used in showing appropriate values of a realvalued variable $w$ of various models. We need information regarding the maximum of the function

$$
f(w)=\left(\begin{array}{c}
n-w \\
d
\end{array}\right)\left(\begin{array}{c}
n-w-d \\
r-s
\end{array}\right)\left(\begin{array}{l}
w \\
s
\end{array}\right)
$$

with various $r$ and $s$ when dealing with possible upper bounds for the size $t$ of various models. Lemma 2.11 and Lemma 2.12 will be used in the simplifications of the bounds $M / v$ and $\ln a$ respectively in the expression $(M / v)(1+\ln a)$ found in the Stein-Lovász theorem (Theorem 2.6).

Lemma 2.9 For any positive integers $n, d, r, s$ with $k=d+r \leq n$ and $1 \leq s \leq r$, the function $f(w)$ in $(*)$ gets its maximum at $w=(n s-(k-s)) / k$.

Proof First note that

$$
f(w)=\left(\begin{array}{c}
n-w \\
k-s
\end{array}\right)\left(\begin{array}{c}
w \\
s
\end{array}\right)\left(\begin{array}{c}
k-s \\
d
\end{array}\right),
$$

and

$$
f(w+1)=\left(\frac{(w+1)(n-w)-(w+1)(k-s)}{(w+1)(n-w)-s(n-w)}\right) f(w) .
$$


Note also that

$$
\frac{(w+1)(n-w)-(w+1)(k-s)}{(w+1)(n-w)-s(n-w)} \begin{cases}\geq 1 & \text { if } w \leq(n s-(k-s)) / k \\ \leq 1 & \text { otherwise. }\end{cases}
$$

Hence $f(w)$ is increasing for $w \leq(n s-(k-s)) / k$, and decreasing otherwise, as required.

By taking $s=r=c$ in $f(w)$, we get the function

$$
g(w)=\left(\begin{array}{l}
w \\
c
\end{array}\right)\left(\begin{array}{l}
n-w \\
k-c
\end{array}\right)
$$

for selectors, and Corollary 2.10 follows immediately.

Corollary 2.10 The function $g(w)$ in $(* *)$ gets its maximum at $w=(n c-(k-c)) / k$.

Lemma 2.11 For any positive integers $n, d, r, s$ with $k=d+r \leq n$ and $1 \leq s \leq r$,

$$
\frac{\prod_{i=0}^{r+d-1}(n-i)}{\prod_{i=0}^{s-1}(n-i k / s) \prod_{j=0}^{k-s-1}(n-j k /(k-s))} \leq 1 .
$$

Proof Without loss of generality, let $s \leq k-s$ and thus $1 \leq k /(k-s) \leq 2 \leq k / s$. To prove this inequality, we will give a bijection

$$
\begin{aligned}
f: & \{0,1, \ldots, r+d-1\} \\
& \rightarrow\{i k / s \mid 0 \leq i \leq s-1\} \cup\{j k /(k-s) \mid 0 \leq j \leq k-s-1\}
\end{aligned}
$$

with the property that $f(t) \leq t$, and hence $(n-t) /(n-f(t)) \leq 1$ for $0 \leq t \leq r+$ $d-1$. Note that the element 0 will be counted twice as $0 \cdot(k / s)$ and $0 \cdot(k /(k-s))$ respectively in the range of the function $f$. Note also that for the case $i+j=t$, if $i k / s=i+i(k-s) / s>t$, then $s /(k-s)<i /(t-i)$ and hence $j k /(k-s)=$ $j(1+s /(k-s))<j(1+i /(t-i))=j(t /(t-i))=t$.

Such a function $f$ is defined recursively as follows. For $0 \leq t \leq 2$, let $f(0)=$ $0 \cdot(k / s), f(1)=0 \cdot(k /(k-s)), f(2)=k /(k-s)$. For $3 \leq t \leq r+d-1$, let $i$ (resp. $j$ ) be the smallest positive integers such that $i k / s$ (resp. $j k /(k-s)) \notin$ $\{f(0), f(1), \ldots, f(t-1)\}$ if they exist, in this case it follows that $i+j=t$.

1. Let $f(t)=i k / s$ if $i k / s \leq t$; otherwise define $f(t)=j k /(k-s)$.

2. For the case $t$ is large and there is no such $i$, note that

$$
\frac{n-s}{n} \leq \frac{n-s-1}{n-k /(k-s)} \leq \cdots \leq \frac{n-r-d+1}{n-k+k /(k-s)}<1,
$$

then $\frac{n-t}{n-(t-s)(k /(k-s))} \leq 1$ for $s \leq t \leq r+d-1$, and define $f(t)=$ $(t-s)(k /(k-s))$. 
Clearly, the function $f$ defined above is a bijection and $f(t) \leq t$ for $0 \leq t \leq$ $r+d-1$, as required.

Lemma 2.12 For any positive integers $n, d, r, s$ with $k=d+r \leq n$ and $1 \leq s \leq r$, let $n^{\prime} \geq n$ be the smallest positive integer such that $w=n^{\prime} s / k$ is an integer, then

1. $\frac{\left(\begin{array}{c}n^{\prime} \\ d\end{array}\right)\left(\begin{array}{c}n^{\prime}-d \\ r\end{array}\right)}{\left(\begin{array}{c}n^{\prime}-w \\ d\end{array}\right)\left(\begin{array}{c}n^{\prime}-w-d \\ r-s\end{array}\right)\left(\begin{array}{c}w \\ s\end{array}\right)} \leq \frac{\left(\frac{k}{s}\right)^{s}\left(\frac{k}{k-s}\right)^{k-s}}{\left(\begin{array}{c}r \\ s\end{array}\right)}$, and

2. $\ln \left(\left(\begin{array}{c}n^{\prime}-w \\ d\end{array}\right)\left(\begin{array}{c}n^{\prime}-w-d \\ r-s\end{array}\right)\left(\begin{array}{c}w \\ s\end{array}\right)\right)<k\left[1+\ln \left(\frac{n}{k}+1\right)\right]+\ln \left(\begin{array}{c}k-s \\ d\end{array}\right)$.

Proof To prove 1, the left hand side can be rewritten as

$$
\frac{\left(\frac{k}{s}\right)^{s}\left(\frac{k}{k-s}\right)^{k-s}}{\left(\begin{array}{l}
r \\
s
\end{array}\right)} \cdot \frac{\prod_{i=0}^{r+d-1}\left(n^{\prime}-i\right)}{\prod_{i=0}^{s-1}\left(n^{\prime}-i k / s\right) \prod_{j=0}^{k-s-1}\left(n^{\prime}-j k /(k-s)\right)} \leq \frac{\left(\frac{k}{s}\right)^{s}\left(\frac{k}{k-s}\right)^{k-s}}{\left(\begin{array}{l}
r \\
s
\end{array}\right)}
$$

by Lemma 2.11. To prove 2, first note that $n^{\prime}<n+k$ for such $n^{\prime}$. By the inequality $\left(\begin{array}{l}a \\ b\end{array}\right) \leq\left(\frac{e a}{b}\right)^{b},\left(\begin{array}{c}n^{\prime}-w \\ d\end{array}\right)\left(\begin{array}{c}n^{\prime}-w-d \\ r-s\end{array}\right)\left(\begin{array}{l}w \\ s\end{array}\right)$ can be rewritten as

$$
\begin{aligned}
\left(\begin{array}{c}
n^{\prime}-w \\
k-s
\end{array}\right)\left(\begin{array}{c}
w \\
s
\end{array}\right)\left(\begin{array}{c}
k-s \\
d
\end{array}\right) & \leq\left(\begin{array}{c}
n^{\prime} \\
k
\end{array}\right)\left(\begin{array}{c}
k-s \\
d
\end{array}\right) \leq\left(\frac{e n^{\prime}}{k}\right)^{k}\left(\begin{array}{c}
k-s \\
d
\end{array}\right) \\
& <e^{k}\left(\frac{n}{k}+1\right)^{k}\left(\begin{array}{c}
k-s \\
d
\end{array}\right) .
\end{aligned}
$$

Therefore,

$$
\begin{aligned}
\ln \left(\left(\begin{array}{c}
n^{\prime}-w \\
d
\end{array}\right)\left(\begin{array}{c}
n^{\prime}-w-d \\
r-s
\end{array}\right)\left(\begin{array}{c}
w \\
s
\end{array}\right)\right) & <\ln \left(e^{k}\left(\frac{n}{k}+1\right)^{k}\left(\begin{array}{c}
k-s \\
d
\end{array}\right)\right) \\
& =k\left[1+\ln \left(\frac{n}{k}+1\right)\right]+\ln \left(\begin{array}{c}
k-s \\
d
\end{array}\right),
\end{aligned}
$$

as required.

The substitutions of $w$ for various subclasses are summarized in the following Table 2.

\section{Extension of the Stein-Lovász theorem}

The Stein-Lovász theorem can be extended from rows with weight at least 1 to the case of rows with weight at least $z \geq 1$. Moreover, the bound can be further improved when $A$ is a matrix with constant row weight and column weight as well.

Theorem 3.1 (Extension of the Stein-Lovász theorem) Let A be a $(0,1)$ matrix of order $N \times M$, and let $v, a, z$ be positive integers. Assume that each row contains at 
Table 2 The substitutions of $w$ for various subclasses

\begin{tabular}{llll}
\hline Types & Parameters & & \\
\hline$d$-disjunct & $s=r=1, z=1$ & $w=(n-d) / k$ & $w=n^{\prime} / k$ \\
$(d ; z]$-disjunct & $s=r=1$ & $w=(n-d) / k$ & $w=n^{\prime} / k$ \\
$(d, r]$-disjunct & $s=r, z=1$ & $w=(n r-d) / k$ & $w=n^{\prime} r / k$ \\
$(d, r ; z]$-disjunct & $s=r$ & $w=(n r-d) / k$ & $w=n^{\prime} r / k$ \\
$(d, r)$-disjunct & $s=1, z=1$ & $w=(n-(k-1)) / k$ & $w=n^{\prime} / k$ \\
$(d, r ; z)$-disjunct & $s=1$ & $w=(n-(k-1)) / k$ & $w=n^{\prime} / k$ \\
$(d, s$ out of $r$-disjunct & $z=1$ & $w=(n s-(k-s)) / k$ & $w=n^{\prime} s / k$ \\
$(d, s$ out of $r ; z]$-disjunct & & $w=(n s-(k-s)) / k$ & $w=n^{\prime} s / k$ \\
$(k, m, n)$-selectors & $c=1, z=1$ & $w=(n-(k-1)) / k$ & $w=n^{\prime} / k$ \\
$(k, m, n ; z)$-selectors & $c=1$ & $w=(n-(k-1)) / k$ & $w=n^{\prime} / k$ \\
$(k, m, c, n)$-selectors & $z=1$ & $w=(n c-(k-c)) / k$ & $w=n^{\prime} c / k$ \\
$(k, m, c, n ; z)$-selectors & & $w=(n c-(k-c)) / k$ & $w=n^{\prime} c / k$ \\
\hline
\end{tabular}

least $v$ ones, and each column at most a ones. Then there exists an $N \times K$ submatrix $C$ with

$$
K \leq z\left(\frac{M}{v-(z-1)}\right)(1+\ln a),
$$

such that each row of $C$ has weight at least $z$. More specifically, if each row of $A$ contains exactly $v$ ones (i.e., A is v-uniform) and each column exactly a ones (i.e., $A$ is a-regular), then the upper bound can be reduced to

$$
K \leq z\left(\frac{M}{v}\right)(1+\ln a) .
$$

The strategy for the proof of Theorem 3.1 is stated as follows:

1. Use the Stein-Lovász theorem to obtain a submatrix $C_{1}$ with each row has weight at least 1 .

2. Choose some columns in the matrix $A \backslash C_{1}$ to combine with the submatrix $C_{1}$ to form a submatrix $C_{2}$ with each row has weight at least 2 .

3. Choose some columns in the matrix $A \backslash C_{2}$ to combine with the submatrix $C_{2}$ to form a submatrix $C_{3}$ with each row has weight at least 3 .

4. Step by step, and finally we obtain the desired submatrix $C=C_{z}$ with each row has weight at least $z$.

Proof A constructive approach for producing $C$ is presented. Let $A_{1}=A$. By the Stein-Lovász theorem, there exists an $N \times M_{1}$ submatrix $C_{1}\left(=B_{1}^{\prime}=B_{1}\right)$ of $A_{1}$ with $M_{1} \leq(M / v)(1+\ln a)$ such that each row of $C_{1}$ has weight at least 1.

The algorithm used in the proof of the Stein-Lovász theorem shows that some rows of $C_{1}$ have weight exactly 1 . Let $R_{1}$ be the set of indices of those rows and let $\left|R_{1}\right|=$ $r_{1}$. Let $A_{2}$ be the submatrix of order $r_{1} \times\left(M-M_{1}\right)$ obtained from $A_{1}$ by deleting the 
submatrix $C_{1}$ and the $k$-th row, $k \notin R_{1}$ as well. Then each row of $A_{2}$ contains at least $v-1$ ones, and each column at most $a$ ones. Again, by the Stein-Lovász theorem, there exists an $r_{1} \times M_{2}$ submatrix $B_{2}^{\prime}$ with $M_{2} \leq\left(\left(M-M_{1}\right) /(v-1)\right)(1+\ln a)$ such that each row of $B_{2}^{\prime}$ has weight at least 1 . Let $B_{2}$ be the matrix of order $N \times M_{2}$ obtained from $B_{2}^{\prime}$ by adding the $k$-th row, $k \notin R_{1}$. Let $C_{2}$ be the matrix of order $N \times\left(M_{1}+M_{2}\right)$ obtained by the union of $B_{1}$ and $B_{2}$. Then $C_{2}$ is a submatrix of $A$ with each row weight at least 2.

Similarly, there exist some rows of $C_{2}$ that have weight exactly 2 . Let $R_{2}$ be the set of indices of those rows and let $\left|R_{2}\right|=r_{2}$. Continue in this way, for $2 \leq i \leq z$ we have

1. $A_{i}$ is a matrix of order $r_{i-1} \times\left(M-\sum_{j=1}^{i-1} M_{j}\right)$, and each row contains at least $v-(i-1)$ ones, and each column at most $a$ ones;

2. $B_{i}^{\prime}$ is an $r_{i-1} \times M_{i}$ submatrix of $A_{i}$ with $M_{i} \leq \frac{M-\sum_{j=1}^{i-1} M_{j}}{v-(i-1)}(1+\ln a)$, and each row has weight at least 1 ;

3. $B_{i}$ is a matrix of order $N \times M_{i}$ obtained from $B_{i}^{\prime}$ by adding the $k$-th row, $k \notin R_{i-1}$;

4. $C_{i}$ is an $N \times \sum_{j=1}^{i} M_{j}$ submatrix of $A$, and each row has weight at least $i$.

Hence, $C=C_{z}$ is the desired submatrix, and

$$
\begin{aligned}
K & =\sum_{j=1}^{z} M_{j}=M_{1}+M_{2}+\cdots+M_{z} \\
& \leq \frac{M}{v}(1+\ln a)+\frac{M-M_{1}}{v-1}(1+\ln a)+\cdots+\frac{M-\sum_{j=1}^{z-1} M_{j}}{v-(z-1)}(1+\ln a) \\
& \leq \frac{M}{v}(1+\ln a)+\frac{M}{v-1}(1+\ln a)+\cdots+\frac{M}{v-(z-1)}(1+\ln a) \\
& \leq z\left(\frac{M}{v-(z-1)}\right)(1+\ln a),
\end{aligned}
$$

thus giving the result.

More specifically, for the case of uniform and regular, using similar argument as above with a minor modification. First we note that $N v=M a$ by counting the number of ones in $A$ in two ways. For $2 \leq i \leq z, A_{i}$ is a matrix of order $r_{i-1} \times$ $\left(M-\sum_{j=1}^{i-1} M_{j}\right)$, and each row contains exactly $v-(i-1)$ ones, and each column at most $a$ ones. Moreover, a lower bound for $\sum_{j=1}^{i-1} M_{j}$ is derived by counting the number of ones in the submatrix $C_{i-1}$ in two ways: each row of $C_{i-1}$ contains at least $i-1$ ones, and each column exactly $a$ ones, thus $N(i-1) \leq\left(\sum_{j=1}^{i-1} M_{j}\right) a$, and hence $(M / v)(i-1) \leq \sum_{j=1}^{i-1} M_{j}$ for $2 \leq i \leq z$. Furthermore,

$$
\begin{aligned}
K & =\sum_{j=1}^{z} M_{j}=M_{1}+M_{2}+\cdots+M_{z} \\
& \leq \frac{M}{v}(1+\ln a)+\frac{M-M_{1}}{v-1}(1+\ln a)+\cdots+\frac{M-\sum_{j=1}^{z-1} M_{j}}{v-(z-1)}(1+\ln a)
\end{aligned}
$$




$$
\begin{aligned}
\leq & \frac{M}{v}(1+\ln a)+\frac{M-M / v}{v-1}(1+\ln a)+\cdots \\
& +\frac{M-(M / v)(z-1)}{v-(z-1)}(1+\ln a) \\
= & z\left(\frac{M}{v}\right)(1+\ln a),
\end{aligned}
$$

thus giving the result.

For the case of uniform and regular, Theorem 3.1 can be restated in the language of hypergraphs as follows:

Corollary 3.2 Let $H=(X, \Gamma)$ be a $v$-uniform and a-regular hypergraph with vertex set $X$ and edge set $\Gamma$, then $\tau_{z}(H) \leq z(|X| / v)(1+\ln a)$.

We conjecture that $\tau_{z}(H) \leq z \tau_{1}(H)$ holds for hypergraphs which are uniform and regular. However, it need not be true in general as shown in the following example. For the hypergraph $H$ with $X=\{1,2,3, \ldots, 8\}$ and $\Gamma=$ $\{\{1,2,3\},\{4,5,6\},\{1,7,8\}\}$. It is easy to see that $\{1,4\}$ is a 1 -cover with minimum size, hence $\tau_{1}(H)=2$. Similarly, $\{1,2,4,5,7\}$ is a 2 -cover with minimum size, hence $\tau_{2}(H)=5$. This shows that $\tau_{2}(H)=5>2 \cdot 2=2 \tau_{1}(H)$.

\section{Some applications of the extended Stein-Lovász theorem}

In this section, the extended Stein-Lovász theorem will be used in dealing the upper bounds for the sizes of $(d, s$ out of $r ; z]$-disjunct matrices (Theorem 4.1) and $(k, m, c, n ; z)$-selectors (Theorem 4.3). It in turn provides a few upper bounds for the sizes in various models with specific parameters as shown in Corollary 4.2 and Corollary 4.4 .

For positive integers $n$ and $d$, let $[n]$ denote the set $\{1,2, \ldots, n\}$ and $\left(\begin{array}{c}{[n]} \\ d\end{array}\right)$ denote the collection of all subsets of $[n]$ with cardinality $d$. For any vector $u \in\{0,1\}^{n}$, the weight $\operatorname{wt}(u)$ of $u$ is the number of coordinates equal to one. Recall that $t(n, d, r, s ; z]$ is the minimum size over all $(d, s$ out of $r ; z]$-disjunct matrices with $n$ columns.

Theorem 4.1 For any positive integers $n, d, r, s$ and $z$, with $1 \leq s \leq r$, if $k=$ $d+r \leq n$, then

$$
\begin{aligned}
t(n, d, r, s ; z]< & \left(z\left(\frac{k}{s}\right)^{s}\left(\frac{k}{k-s}\right)^{k-s} /\left(\begin{array}{l}
r \\
s
\end{array}\right)\right)\left\{1+k\left[1+\ln \left(\frac{n}{k}+1\right)\right]\right. \\
& \left.+\ln \left(\begin{array}{c}
k-s \\
d
\end{array}\right)\right\} .
\end{aligned}
$$


Proof For $s \leq w \leq n-d$, let $A$ be the binary matrix of order $\left[\left(\begin{array}{c}n \\ d\end{array}\right)\left(\begin{array}{c}n-d \\ r\end{array}\right)\right] \times\left(\begin{array}{c}n \\ w\end{array}\right)$ with rows and columns indexed by $\left\{(D, R) \mid D \in\left(\begin{array}{c}{[n]} \\ d\end{array}\right), R \in\left(\begin{array}{c}{[n]} \\ r\end{array}\right)\right.$ with $\left.D \cap R=\emptyset\right\}$ and $U=\left\{u \mid u \in\{0,1\}^{n}, \mathrm{wt}(u)=w\right\}$, respectively. The entry of $A$ at the row indexed by the pair $(D, R)$ and the column indexed by the vector $u \in U$ is 1 if the entries of $u$ over $D$ are all zero and at least $s$ entries of $u$ over $R$ are one; and 0 otherwise.

Observe that each row of $A$ has weight

$$
v=\sum_{j=s}^{\min (r, w)}\left(\begin{array}{l}
r \\
j
\end{array}\right)\left(\begin{array}{c}
n-(d+r) \\
w-j
\end{array}\right)
$$

and each column of $A$ has weight

$$
a=\left(\begin{array}{c}
n-w \\
d
\end{array}\right) \sum_{j=s}^{\min (r, w)}\left(\begin{array}{c}
n-w-d \\
r-j
\end{array}\right)\left(\begin{array}{l}
w \\
j
\end{array}\right)
$$

By the extended Stein-Lovász theorem, there exists a submatrix $M$ of $A$ of order $\left[\left(\begin{array}{c}n \\ d\end{array}\right)\left(\begin{array}{c}n-d \\ r\end{array}\right)\right] \times t$ with each row has weight at least $z$, where

$$
t \leq z\left(\left(\begin{array}{l}
n \\
w
\end{array}\right) / v\right)\{1+\ln a\}=z\left(\left(\begin{array}{l}
n \\
d
\end{array}\right)\left(\begin{array}{c}
n-d \\
r
\end{array}\right) / a\right)\{1+\ln a\} .
$$

Note that the equality is obtained by counting the number of ones in $A$ in two ways.

Let $M^{\prime}=\left(m_{u i}^{\prime}\right)$ be a $t \times n$ matrix with rows indexed by the column indices of $M$ and columns indexed by $[n]$ such that

$$
m_{u i}^{\prime}= \begin{cases}1 & \text { if the } i \text { th coordinate of the vector } u \text { is } 1 \\ 0 & \text { otherwise. }\end{cases}
$$

Then it is straightforward to show that $M^{\prime}$ is a $(d, s$ out of $r ; z]$-disjunct matrix, and thus

$$
t(n, d, r, s ; z] \leq z\left(\left(\begin{array}{l}
n \\
d
\end{array}\right)\left(\begin{array}{c}
n-d \\
r
\end{array}\right) / a\right)\{1+\ln a\} \leq z\left(\left(\begin{array}{l}
n \\
d
\end{array}\right)\left(\begin{array}{c}
n-d \\
r
\end{array}\right) / a^{\prime}\right)\left\{1+\ln a^{\prime}\right\},
$$

where $a^{\prime}=\left(\begin{array}{c}n-w \\ d\end{array}\right)\left(\begin{array}{c}n-w-d \\ r-s\end{array}\right)\left(\begin{array}{l}w \\ s\end{array}\right)$.

Let $n^{\prime} \geq n$ be the smallest positive integer such that $w=n^{\prime} s / k$ is an integer. By Lemma 2.12, we have

$$
\frac{\left(\begin{array}{l}
n^{\prime} \\
d
\end{array}\right)\left(\begin{array}{c}
n^{\prime}-d \\
r
\end{array}\right)}{\left(\begin{array}{c}
n^{\prime}-w \\
d
\end{array}\right)\left(\begin{array}{c}
n^{\prime}-w-d \\
r-s
\end{array}\right)\left(\begin{array}{l}
w \\
s
\end{array}\right)} \leq \frac{\left(\frac{k}{s}\right)^{s}\left(\frac{k}{k-s}\right)^{k-s}}{\left(\begin{array}{l}
r \\
s
\end{array}\right)}
$$

and

$$
\ln \left(\left(\begin{array}{c}
n^{\prime}-w \\
d
\end{array}\right)\left(\begin{array}{c}
n^{\prime}-w-d \\
r-s
\end{array}\right)\left(\begin{array}{c}
w \\
s
\end{array}\right)\right)<k\left[1+\ln \left(\frac{n}{k}+1\right)\right]+\ln \left(\begin{array}{c}
k-s \\
d
\end{array}\right) .
$$


Therefore,

$$
\begin{aligned}
t(n, d, r, s ; z] & \leq t\left(n^{\prime}, d, r, s ; z\right] \\
& \leq \frac{z\left(\begin{array}{c}
n^{\prime} \\
d
\end{array}\right)\left(\begin{array}{c}
n^{\prime}-d \\
r
\end{array}\right)}{\left(\begin{array}{c}
n^{\prime}-w \\
d
\end{array}\right)\left(\begin{array}{c}
n^{\prime}-w-d \\
r-s
\end{array}\right)\left(\begin{array}{c}
w \\
s
\end{array}\right)}\left\{1+\ln \left[\left(\begin{array}{c}
n^{\prime}-w \\
d
\end{array}\right)\left(\begin{array}{c}
n^{\prime}-w-d \\
r-s
\end{array}\right)\left(\begin{array}{l}
w \\
s
\end{array}\right)\right]\right\} \\
& <\frac{z\left(\frac{k}{s}\right)^{s}\left(\frac{k}{k-s}\right)^{k-s}}{\left(\begin{array}{c}
r \\
s
\end{array}\right)}\left\{1+k\left[1+\ln \left(\frac{n}{k}+1\right)\right]+\ln \left(\begin{array}{c}
k-s \\
d
\end{array}\right)\right\}
\end{aligned}
$$

as required.

As a consequence, upper bounds for the sizes of various situations are summarized in the following corollary.

\section{Corollary 4.2 (Lee 2009)}

1. If $k=d+1 \leq n$, then

$$
\begin{gathered}
t(n, d)=t(n, d, 1,1 ; 1]<k\left(\frac{k}{d}\right)^{d}\left\{1+k\left[1+\ln \left(\frac{n}{k}+1\right)\right]\right\} \\
t(n, d ; z]=t(n, d, 1,1 ; z]<z k\left(\frac{k}{d}\right)^{d}\left\{1+k\left[1+\ln \left(\frac{n}{k}+1\right)\right]\right\} .
\end{gathered}
$$

2. If $k=d+r \leq n$, then

$$
\begin{aligned}
t(n, d, r] & =t(n, d, r, r ; 1]<\left(\frac{k}{r}\right)^{r}\left(\frac{k}{d}\right)^{d}\left\{1+k\left[1+\ln \left(\frac{n}{k}+1\right)\right]\right\} \\
t(n, d, r ; z] & =t(n, d, r, r ; z]<z\left(\frac{k}{r}\right)^{r}\left(\frac{k}{d}\right)^{d}\left\{1+k\left[1+\ln \left(\frac{n}{k}+1\right)\right]\right\} ; \\
t(n, d, r) & =t(n, d, r, 1 ; 1] \\
& <\frac{k}{r}\left(\frac{k}{k-1}\right)^{k-1}\left\{1+k\left[1+\ln \left(\frac{n}{k}+1\right)\right]+\ln \left(\begin{array}{c}
k-1 \\
d
\end{array}\right)\right\} ; \\
t(n, d, r ; z) & =t(n, d, r, 1 ; z] \\
& <z \frac{k}{r}\left(\frac{k}{k-1}\right)^{k-1}\left\{1+k\left[1+\ln \left(\frac{n}{k}+1\right)\right]+\ln \left(\begin{array}{c}
k-1 \\
d
\end{array}\right)\right\} \\
t(n, d, r, s] & =t(n, d, r, s ; 1] \\
& <\frac{\left(\frac{k}{s}\right)^{s}\left(\frac{k}{k-s}\right)^{k-s}}{\left(\begin{array}{c}
r \\
s
\end{array}\right)}\left\{1+k\left[1+\ln \left(\frac{n}{k}+1\right)\right]+\ln \left(\begin{array}{c}
k-s \\
d
\end{array}\right)\right\} .
\end{aligned}
$$

Following similar arguments in De Bonis et al. (2005) and De Bonis (2008) with a minor modification, the upper bound for the sizes of $(k, m, c, n ; z)$-selectors is 
given below. Recall that $t_{s}(k, m, c, n ; z)$ is the minimum size over all $(k, m, c, n ; z)$ selectors.

Theorem 4.3 For $b=\left(\begin{array}{l}k \\ c\end{array}\right)$,

$$
\begin{aligned}
t_{s}(k, m, c, n ; z)< & \frac{(b-m+1)(z-1)+1}{b-m+1}\left(\frac{k}{c}\right)^{c}\left(1+\frac{1}{k-c}\right)^{k-c} \\
& \times\left\{1+k\left[1+\ln \left(\frac{n}{k}+1\right)\right]+\ln \left(\begin{array}{c}
b-1 \\
b-m
\end{array}\right)\right\} .
\end{aligned}
$$

Proof For $c \leq w \leq n-k+c$, let $X=\left\{x \in\{0,1\}^{n} \mid \operatorname{wt}(x)=w\right\}$ and $U=\{u \in$ $\left.\{0,1\}^{k} \mid \operatorname{wt}(u)=c\right\}$. Note that $|U|=b$. Moreover, for any $A \subseteq U$ of size $r, 1 \leq r \leq b$, and any set $S \in\left(\begin{array}{c}{[n]} \\ k\end{array}\right)$, define $E_{A, S}=\left\{x \in X:\left.x\right|_{S} \in A\right\}$.

Let $M$ be the binary matrix of order $\left[\left(\begin{array}{c}b \\ b-m+1\end{array}\right)\left(\begin{array}{l}n \\ k\end{array}\right)\right] \times\left(\begin{array}{l}n \\ w\end{array}\right)$ with rows and columns in-

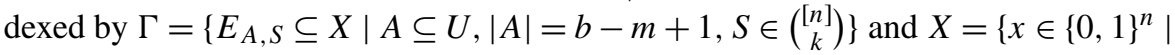
$\operatorname{wt}(x)=w\}$, respectively. The entry of $M$ at the row indexed by the set $E_{A, S}$ and the column indexed by the vector $x \in X$ is 1 if $x \in E_{A, S}$; and 0 otherwise.

Observe that each row of $M$ has weight

$$
v=\left(\begin{array}{c}
b-m+1 \\
1
\end{array}\right)\left(\begin{array}{l}
n-k \\
w-c
\end{array}\right)
$$

and each column of $M$ has weight

$$
a=\left(\begin{array}{l}
w \\
c
\end{array}\right)\left(\begin{array}{c}
n-w \\
k-c
\end{array}\right)\left(\begin{array}{c}
b-1 \\
(b-m+1)-1
\end{array}\right) .
$$

By the extended Stein-Lovász theorem, there exists a submatrix $M^{\prime}$ of $M$ of order $\left[\left(\begin{array}{c}b \\ b-m+1\end{array}\right)\left(\begin{array}{l}n \\ k\end{array}\right)\right] \times t$ with each row has weight at least $f=(b-m+1)(z-1)+1$, where

$$
t \leq f\left(\left(\begin{array}{l}
n \\
w
\end{array}\right) / v\right)\{1+\ln a\}=f\left(\left(\begin{array}{c}
b \\
b-m+1
\end{array}\right)\left(\begin{array}{l}
n \\
k
\end{array}\right) / a\right)\{1+\ln a\} .
$$

Note that the equality is obtained by counting the number of ones in $M$ in two ways.

Let $M^{*}=\left(m_{x i}^{*}\right)$ be a $t \times n$ matrix with rows indexed by the column indices of $M^{\prime}$ and columns indexed by $[n]$ such that

$$
m_{x i}^{*}= \begin{cases}1 & \text { if the } i \text { th coordinate of the vector } x \text { is } 1 \\ 0 & \text { otherwise. }\end{cases}
$$

Then it suffices to show that $M^{*}$ is a $(k, m, c, n ; z)$-selector, that is, any submatrix of $k$ arbitrary columns of $M^{*}$ contains $z$ disjoint submatrices of order $m \times k$ such that in each of them the $m$ rows are all distinct and each row has exactly $c$ entries equal to 1 . 
Let $x_{1}, x_{2}, \ldots, x_{t}$ be the $t$ rows of $M^{*}$ and let $T=\left\{x_{1}, x_{2}, \ldots, x_{t}\right\}$. Suppose contradictorily that there exists a set $S \in\left(\begin{array}{c}{[n]} \\ k\end{array}\right)$ such that the submatrix $\left.M^{*}\right|_{S}$ of $M^{*}$ contains at most $z-1$ disjoint submatrices of order $m \times k$ such that in each of them the $m$ rows are all distinct and each row has exactly $c$ entries equal to 1 . Moreover, $\left.M^{*}\right|_{S}$ contains another disjoint submatrix with at most $m-1$ distinct rows with weight exactly $c$. Let $u_{j_{1}}, u_{j_{2}}, \ldots, u_{j_{q}}$ be such rows, with $q \leq m-1$; let $A$ be any subset of $U \backslash\left\{u_{j_{1}}, u_{j_{2}}, \ldots, u_{j_{q}}\right\}$ of cardinality $|A|=b-m+1$, then we have $\left|T \cap E_{A, S}\right|<(b-m+1)(z-1)+1$, contradicting the fact that $M^{\prime}$ is a matrix of $\operatorname{order}\left[\left(\begin{array}{c}b \\ b-m+1\end{array}\right)\left(\begin{array}{l}n \\ k\end{array}\right)\right] \times t$ with each row has weight at least $f=(b-m+1)(z-1)+1$. Hence we have

$$
t_{s}(k, m, c, n ; z) \leq f\left(\left(\begin{array}{c}
b \\
b-m+1
\end{array}\right)\left(\begin{array}{l}
n \\
k
\end{array}\right) / a\right)\{1+\ln a\} .
$$

Let $n^{\prime} \geq n$ be the smallest positive integer such that $w=n^{\prime} c / k$ is an integer. By taking $s=r=c$ in Lemma 2.12, we have

$$
\begin{aligned}
\frac{\left(\begin{array}{c}
b \\
b-m+1
\end{array}\right)\left(\begin{array}{c}
n^{\prime} \\
k
\end{array}\right)}{\left(\begin{array}{c}
w \\
c
\end{array}\right)\left(\begin{array}{c}
n^{\prime}-w \\
k-c
\end{array}\right)\left(\begin{array}{c}
b-1 \\
b-m
\end{array}\right)} & =\frac{1}{b-m+1} \cdot \frac{\left(\begin{array}{c}
k \\
c
\end{array}\right)\left(\begin{array}{c}
n^{\prime} \\
k
\end{array}\right)}{\left(\begin{array}{c}
w \\
c
\end{array}\right)\left(\begin{array}{c}
n^{\prime}-w \\
k-c
\end{array}\right)} \\
& \leq \frac{1}{b-m+1}\left(\frac{k}{c}\right)^{c}\left(1+\frac{1}{k-c}\right)^{k-c}
\end{aligned}
$$

and

$$
\ln \left[\left(\begin{array}{l}
w \\
c
\end{array}\right)\left(\begin{array}{c}
n^{\prime}-w \\
k-c
\end{array}\right)\left(\begin{array}{c}
b-1 \\
b-m
\end{array}\right)\right]<k\left[1+\ln \left(\frac{n}{k}+1\right)\right]+\ln \left(\begin{array}{c}
b-1 \\
b-m
\end{array}\right) .
$$

Therefore,

$$
\begin{aligned}
t_{S}(k, m, c, n ; z) \leq & t_{S}\left(k, m, c, n^{\prime} ; z\right) \\
\leq & \frac{[(b-m+1)(z-1)+1]\left(\begin{array}{c}
b \\
b-m+1
\end{array}\right)\left(\begin{array}{c}
n^{\prime} \\
k
\end{array}\right)}{\left(\begin{array}{c}
w \\
c
\end{array}\right)\left(\begin{array}{c}
n^{\prime}-w \\
k-c
\end{array}\right)\left(\begin{array}{c}
b-1 \\
b-m
\end{array}\right)} \\
& \times\left\{1+\ln \left[\left(\begin{array}{c}
w \\
c
\end{array}\right)\left(\begin{array}{c}
n^{\prime}-w \\
k-c
\end{array}\right)\left(\begin{array}{c}
b-1 \\
b-m
\end{array}\right)\right]\right\} \\
< & \frac{(b-m+1)(z-1)+1}{b-m+1}\left(\frac{k}{c}\right)^{c}\left(1+\frac{1}{k-c}\right)^{k-c} \\
& \times\left\{1+k\left[1+\ln \left(\frac{n}{k}+1\right)\right]+\ln \left(\begin{array}{c}
b-1 \\
b-m
\end{array}\right)\right\}
\end{aligned}
$$

as required.

As a consequence, upper bounds for the sizes of various situations are summarized in the following corollary. 
Corollary 4.4 (Lee 2009)

$$
\begin{aligned}
t_{S}(k, m, n)= & t_{s}(k, m, 1, n ; 1) \\
< & \frac{k}{k-m+1}\left(1+\frac{1}{k-1}\right)^{k-1}\left\{1+k\left[1+\ln \left(\frac{n}{k}+1\right)\right]\right. \\
& \left.+\ln \left(\begin{array}{c}
k-1 \\
k-m
\end{array}\right)\right\} . \\
t_{S}(k, m, n ; z)= & t_{s}(k, m, 1, n ; z) \\
< & \frac{k[(k-m+1)(z-1)+1]}{k-m+1}\left(1+\frac{1}{k-1}\right)^{k-1} \\
& \times\left\{1+k\left[1+\ln \left(\frac{n}{k}+1\right)\right]+\ln \left(\begin{array}{l}
k-1 \\
k-m
\end{array}\right)\right\} . \\
t_{S}(k, m, c, n)= & t_{s}(k, m, c, n ; 1) \\
< & \frac{1}{b-m+1}\left(\frac{k}{c}\right)^{c}\left(1+\frac{1}{k-c}\right)^{k-c} \\
& \times\left\{1+k\left[1+\ln \left(\frac{n}{k}+1\right)\right]+\ln \left(\begin{array}{l}
b-1 \\
b-m
\end{array}\right)\right\} .
\end{aligned}
$$

\section{Concluding remarks}

In this paper, we derive the extended Stein-Lovász theorem to deal with more combinatorial structures. From the strategy of the proof in Theorem 3.1, it is easy to see that the extended Stein-Lovász theorem also provides an algorithmic way to deal with the existence of good coverings, and thus offers bounds related to some combinatorial structures. Note that most of these bounds are roughly the same as those derived by the basic probabilistic methods including the Lovász Local Lemma (Yeh 2002; Yu 2007). Thus, due to its constructive nature, the Stein-Lovász theorem can be regarded as a de-randomized algorithm for the probabilistic methods. The relationship between the (extended) Stein-Lovász theorem and the Lovász Local Lemma deserves further study.

Acknowledgements The author would like to thank the anonymous referees for careful reading and valuable suggestions on presentation of the material.

\section{References}

Cheng Y, Du DZ (2008) New constructions of one- and two-stage pooling designs. J Comput Biol 15:195205

Chen HB, Fu HL, Hwang FK (2008) An upper bound of the number of tests in pooling designs for the error-tolerant complex model. Opt Lett 2:425-431

Cohen G, Litsyn S, Zemor G (1996) On greedy algorithms in coding theory. IEEE Trans Info Theory 42(6):2053-2057 
De Bonis A (2008) New combinatorial structures with applications to efficient group testing with inhibitors. J Comb Optim 15:77-94

De Bonis A, Gasieniec L, Vaccaro U (2005) Optimal two-state algorithms for group testing problems. SIAM J Comput 34(5):1253-1270

Deng D, Zhang Y, Li PC, van Rees GHJ (2011) The Stein-Lovász theorem and its application to some combinatorial arrays. J Combin Math Combin Comput 77:17-31

Fulkerson DR, Ryser HJ (1963) Width sequences for special classes of (0,1)-matrices. Canad J Math $15: 371-396$

Lee GS (2009) An extension of Stein-Lovász theorem and some of its applications. Master thesis, Department of Applied Mathematics, National Chiao-Tung University, Hsinchu, Taiwan

Lovász L (1975) On the ratio of optimal integral and fractional covers. Discrete Math 13:383-390

Stein SK (1974) Two combinatorial covering problems. J Comb Theory, Ser A 16:391-397

Yeh HG (2002) $d$-disjunct matrices: bound and Lovász local lemma. Discrete Math 253:97-107

Yu KA (2007) Applications of the Lovász local lemma to pooling designs. Master thesis, Department of Applied Mathematics, National Chiao-Tung University, Hsinchu, Taiwan 